\title{
Assessing Quality of Life in Oncology Practice: First Results from a Pilot Study
}

\section{INTRODUCTION}

Cancer disease, especially when in advanced stages, brings with it a multiplicity of symptoms amenable to cause great suffering and profoundly compromise quality of life (QoL). Therefore, multidisciplinary strategies which comprise the multidimensionality of the person and that focus in promoting QoL are essential to assure a needs' based provision of care. Health related QoL (HRQL) is an increasingly approached theme in Oncology, simultaneously with diagnostic and therapeutic innovations that encompass patient-centered interventions. Patient-reported Outcomes (PROs) allow to the clinical team to acquire reliable information about the patient's perspective of wellbeing, needs, symptoms and toxicities, among others, allowing for a better communication and discussion of patient's concerns and desires. PROs have been implemented and adapted to clinical practice showing promising results. However, replication of these programs is mandatory, in order to strengthen clinical evidence on the impact of these measures in decision-making processes and in the way we approach cancer patients is mandatory for the improvement of QoL and survival.

\section{RESULTS}

\begin{tabular}{|c|c|c|c|}
\hline \multicolumn{2}{|l|}{ Gender } & \multirow{2}{*}{\multicolumn{2}{|c|}{ Tumor staging }} \\
\hline Male & $11(29.7 \%)$ & & \\
\hline Female & $26(70.3 \%)$ & $|-I|$ & $7(19 \%)$ \\
\hline Age, mean & $66(+-14.3)$ & III-IV & $30(81 \%)$ \\
\hline (range), years & $27-83$ & \multicolumn{2}{|l|}{ Active tumor-directed therapy } \\
\hline Tumour Location & & Yes & $25(68 \%)$ \\
\hline Esophagus, Stomach & $9(24 \%)$ & No & $12(32 \%)$ \\
\hline Colorectal and Anal & $12(32.4 \%)$ & \multicolumn{2}{|l|}{ ECOG/Performance Status } \\
\hline Breast & $8(21.6 \%)$ & 0 & $15(40.5 \%)$ \\
\hline Gynecological & $1(2.7 \%)$ & 1 & $9(24.3 \%)$ \\
\hline Pancreas, Biliary tract, Liver & $5(13.5 \%)$ & 2 & $6(16.2 \%)$ \\
\hline Genitourinary & $2(5.4 \%)$ & 3 & $7(18.9 \%)$ \\
\hline
\end{tabular}

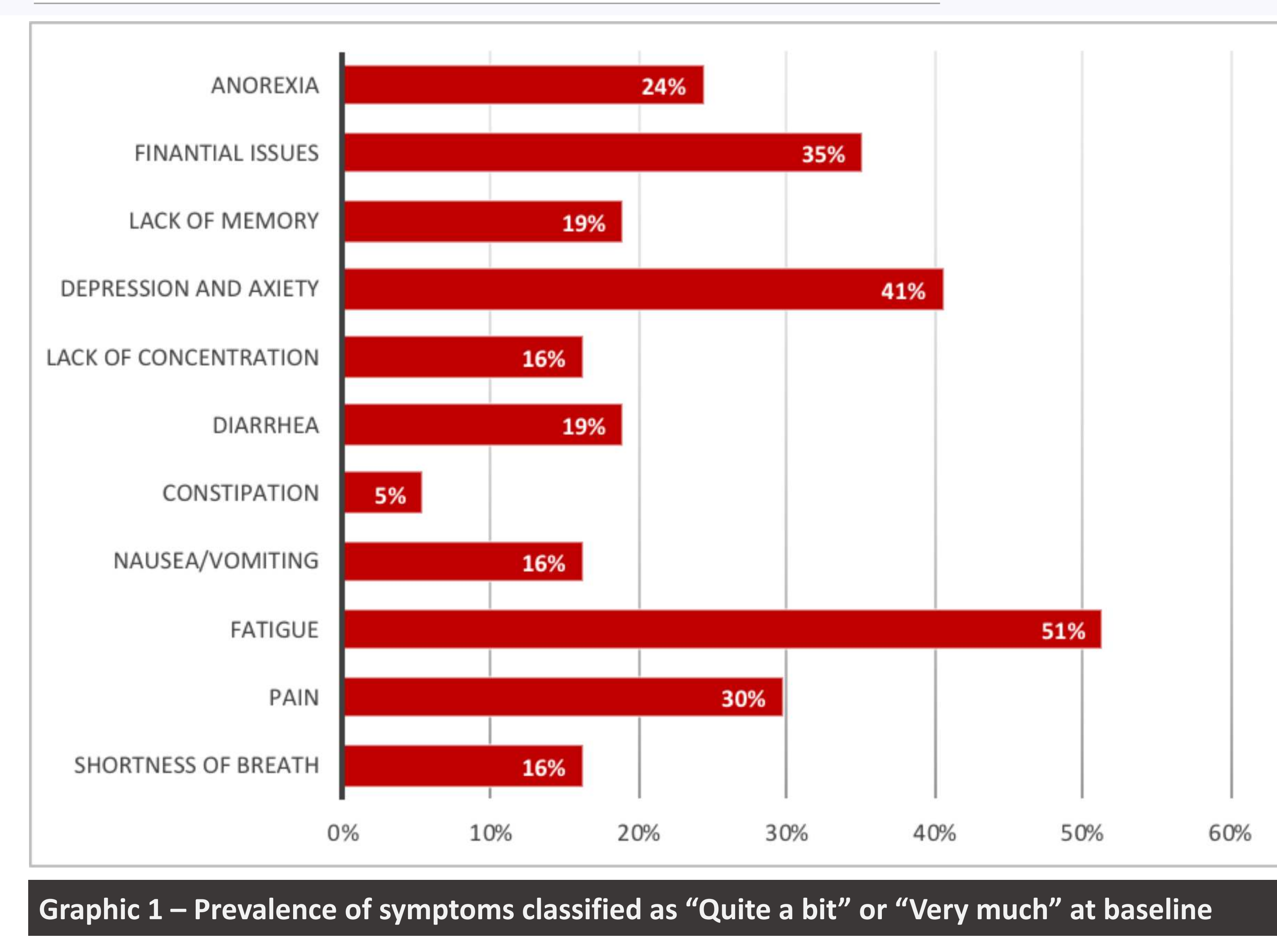

In $29(78 \%)$ patients were identified issues otherwise not verbally referred.

In all cases of improvement in symptom scores, a medical intervention was undertaken.

\section{OBJECTIVES}

Our main goals were to assess feasibility of routine measurement of HRQoL through PRO measures in oncology practice. Additionally, we aimed to understand symptom prevalence, changes over time and to analyze its predictive use, as well as to understand how this information could impact on facilitatin physician-patient communication.

\section{METHODS}

We've implemented a program for systematic and standardized measurement of PROs. This is a prospective, descriptive study, conducted in a tertiary central hospital. Here, we report first results. From January to June 2018, cancer patients were asked to complete the European Organization for Research and Treatment of Cancer Quality of Life Questionnaire-Core 30 (QLQ-C30 version 3.0) in consecutive outpatient visits, delivered to the physician prior to each consultation. Data regarding clinicians attitudes were provided through electronic medical records.

Data analysis using descriptive statistics, chi-square test, kruskall-wallis test, two sample t-test and univariate logistic regression.

\section{Table 3 - Univariate Logistic Regression for improvement in symptom scores \\ Ge \\ Gender Variable \\ Female \\ Male (reference) \\ Metastatic Disease \\ Performance Status \\ Active tumor-directed therapy

\begin{tabular}{|c|c|c|}
\hline OR & Cl 95\% & p-value \\
\hline 1,17 & $0,13-10,22$ & 0,02 \\
\hline 0,67 & $0,05-8,64$ & 0,1 \\
\hline 1,06 & $0,42-2,67$ & 0,01 \\
\hline 1,13 & $0,08-16,31$ & 0,01 \\
\hline 1 & $0,02-1,71$ & 0,00 \\
\hline 0,89 & $0,06-12,88$ & 0,01 \\
\hline
\end{tabular} \\ At 3-month follow-up, 6 (16\%) patient had died. Worsen of symptoms was correlated with bigger odds of dying. \\ Worsening of both scores preceded in all cases evidence of cancer progression (mean of 34 days, SD +- 8).}

In functional scores,

when the difference

is positive, it means

there was an

improvement

So, overall functiona

scores improved,

particularly

emotional and

cognitive, but social

and physical

functioning worsen.

$$
\text { functioning worsen. }
$$

In symptom scores, when the difference is positive, it means there was a worsening of symptoms.

\begin{tabular}{|c|c|c|c|c|c|}
\hline \multirow{7}{*}{$\begin{array}{l}\text { functional scores, } \\
\text { hen the difference } \\
\text { positive, it means } \\
\text { there was an } \\
\text { improvement. } \\
\text { overall functional } \\
\text { scores improved, } \\
\text { particularly } \\
\text { emotional and } \\
\text { gnitive, but social } \\
\text { and physical } \\
\text { nctioning worsen. }\end{array}$} & $\begin{array}{l}\text { QLQ-c30 } \\
\text { domains }\end{array}$ & $\begin{array}{c}\text { Cronbach } \\
\text { alpha }\end{array}$ & Baseline & $\begin{array}{c}2 \text { months post } \\
\text { baseline }\end{array}$ & Difference \\
\hline & $\begin{array}{l}\text { Functional } \\
\text { scores }\end{array}$ & 0.88 & $\begin{array}{c}70.6 \text { (CI 95\% } \\
64.6-76.6)\end{array}$ & $\begin{array}{c}72.8 \text { (CI 95\% } \\
61.5-84.0)\end{array}$ & 2,2 \\
\hline & $\begin{array}{l}\text { Physical } \\
\text { functioning }\end{array}$ & & 70.1 & 66.3 & $-3,8$ \\
\hline & $\begin{array}{c}\text { Role } \\
\text { functioning }\end{array}$ & & 68.9 & 68.6 & $-0,3$ \\
\hline & $\begin{array}{l}\text { Emotional } \\
\text { functioning }\end{array}$ & & 70.9 & 72.1 & 1,2 \\
\hline & $\begin{array}{r}\text { Cognitive } \\
\text { functioning }\end{array}$ & & 76.6 & 78.4 & 1,8 \\
\hline & $\begin{array}{c}\text { Social } \\
\text { functioning }\end{array}$ & & 66.7 & 50.3 & $-16,4$ \\
\hline \multirow{9}{*}{$\begin{array}{l}\text { n symptom scores, } \\
\text { vhen the difference } \\
\text { s positive, it means } \\
\text { there was a } \\
\text { worsening of } \\
\text { symptoms. }\end{array}$} & Symptoms scores & 0.81 & $\begin{array}{c}27.7(\mathrm{Cl} 195 \% \\
22.1-33.4) \\
\end{array}$ & $\begin{array}{c}28.0(\mathrm{Cl} 195 \% \\
17.4-38.7) \\
\end{array}$ & 0,3 \\
\hline & Fatigue & & 41.7 & 9.8 & $-31,9$ \\
\hline & Nausea/vomiting & & 14.0 & 33.3 & 19,3 \\
\hline & Pain & & 35.1 & 15.7 & $-19,4$ \\
\hline & Dyspnea & & 17.1 & 33.3 & 16,2 \\
\hline & Insomnia & & 33.3 & 33.3 & 0 \\
\hline & Appetite loss & & 26.1 & 5.9 & $-20,2$ \\
\hline & Constipation & & 26.1 & 33.3 & 7,2 \\
\hline & Diarrhea & & 20.7 & 5.9 & $-14,8$ \\
\hline \multirow{2}{*}{$\begin{array}{l}\text { Overall, global } \\
\text { health worsen }\end{array}$} & $\begin{array}{l}\text { Financial } \\
\text { Difficulties }\end{array}$ & & 35.1 & 37.3 & 2,2 \\
\hline & Global Health & 0.90 & 57.4 & 48 & $-9,4$ \\
\hline
\end{tabular}

In following visits $(17,44 \%)$, mean deterioration in functional and symptom scores were -21.1 [Cl 95\% 40.5 to -5.5$]$ and 16.6 [Cl 95\% 4.5-29.5].

Functional scores improved in 12 patients (71\%) (10.7, Cl95\% 5.1-16.2), and symptom scores in 11 (65\%) (-12.0, Cl 95\% -17.4 to -7.8). Improvement - Worse

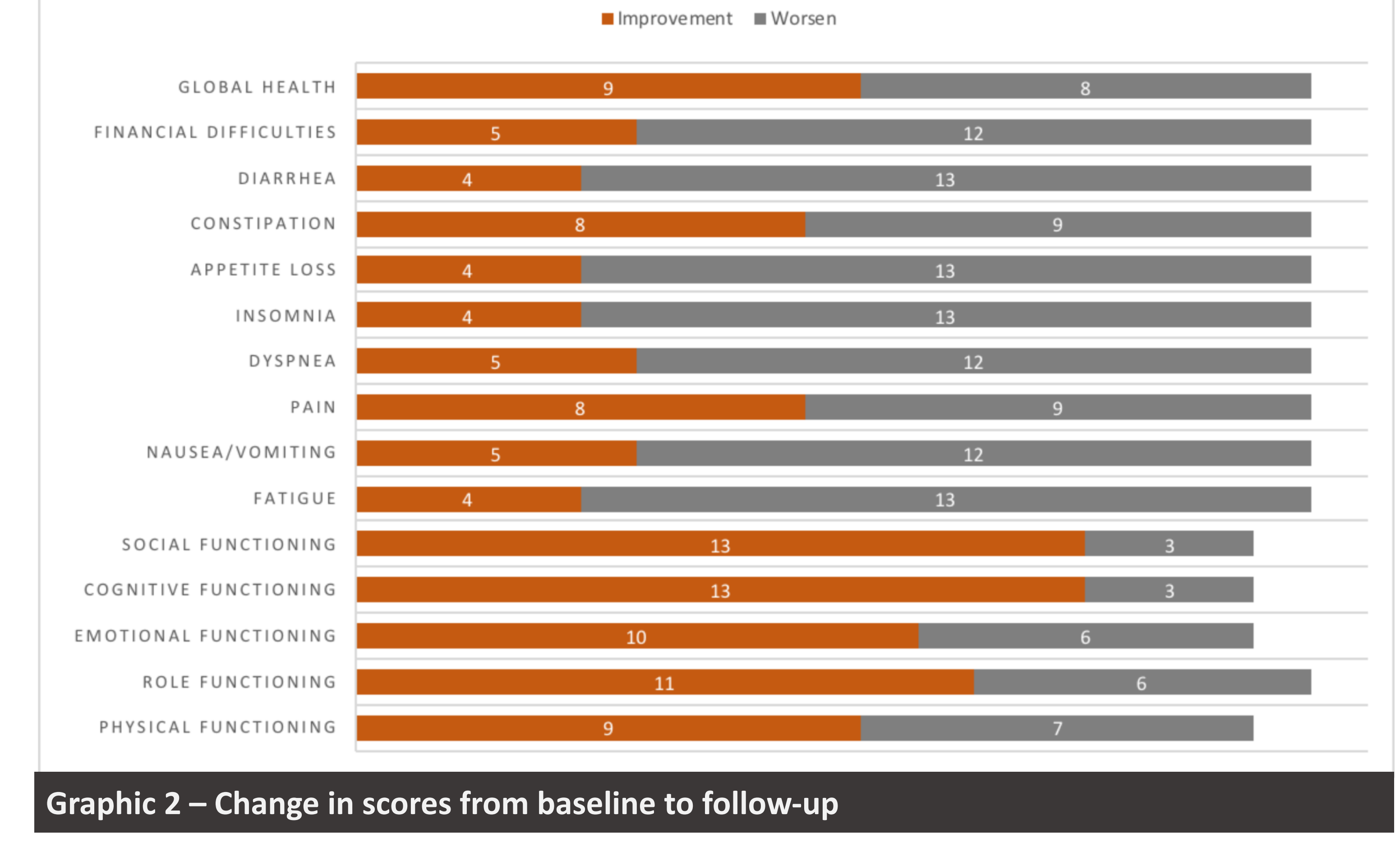

\section{DISCUSSION/CONCLUSIONS}

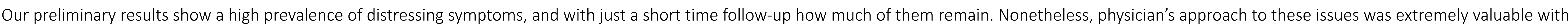

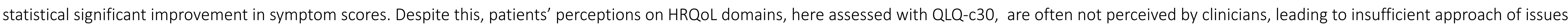

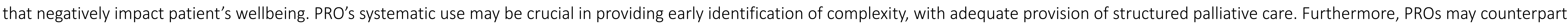

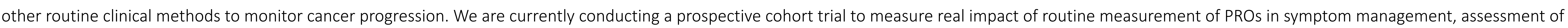
toxicities, in reducing emergency visits, hospital admissions and mortality, which will hopefully reflect into better QoL and survival. 